\title{
Low tidal volume ventilation is associated with reduced mortality in HIV-infected patients with acute lung injury
}

\author{
J L Davis, ${ }^{1}$ A Morris, ${ }^{2}$ R H Kallet, ${ }^{3}$ K Powell, ${ }^{4}$ A S Chi, ${ }^{5}$ M Bensley, ${ }^{6}$ J M Luce, ${ }^{1}$ \\ L Huang ${ }^{1,6}$
}

${ }^{1}$ Division of Pulmonary and Critical Care Medicine, San Francisco General Hospital, University of California, San Francisco, California, USA; ${ }^{2}$ Division of Pulmonary, Allergy, and Critical Care Medicine University of Pittsburgh, Pittsburgh, Pennsylvania, USA;

${ }^{3}$ Department of Anesthesia, San Francisco General Hospital, University of California, San Francisco, California, USA;

${ }^{4}$ Department of Medicine, San Francisco General Hospital, University of California, San Francisco, California, USA;

${ }^{5}$ Division of Pulmonary \& Critical Care Medicine, Boston University, Boston, Massachusetts, USA; ${ }^{6}$ HIV/ AIDS Division, San Francisco General Hospital, University of California, San Francisco,

California, USA

Correspondence to: Dr J L Davis, San Francisco General Hospital, 1001 Potrero Avenue, Room SK1, San Francisco, CA 94110, USA; lucian.davis@ucsf.edu

Received 12 January 2008 Accepted 29 April 2008 Published Online First 5 June 2008

\begin{abstract}
Background: Respiratory failure remains the leading indication for admission to the intensive care unit (ICU) and a leading cause of death for HIV-infected patients in spite of overall improvements in ICU mortality. It is unclear if these improvements are due to combination antiretroviral therapy, low tidal volume ventilation for acute lung injury, or both. A study was undertaken to identify therapies and clinical factors associated with mortality in acute lung injury among HIV-infected patients with respiratory failure in the period 1996-2004. A secondary aim was to compare mortality before and after introduction of a low tidal volume ventilation protocol in 2000.
\end{abstract}

Methods: A retrospective cohort study was performed of 148 consecutive HIV-infected adults admitted to the ICU at San Francisco General Hospital with acute lung injury requiring mechanical ventilation. Demographic and clinical information including data on mechanical ventilation was abstracted from medical records and analysed by multivariate analysis using logistic regression.

Results: In-hospital mortality was similar before and after introduction of a low tidal volume ventilation protocol, although the study was not powered to exclude a clinically significant difference (risk difference $-5.4 \%$, $95 \% \mathrm{Cl}-21 \%$ to $11 \%, \mathrm{p}=0.51)$. Combination antiretroviral therapy was not clearly associated with mortality, except in patients with Pneumocystis pneumonia. Among all those with acute lung injury, lower tidal volume was associated with decreased mortality (adjusted odds ratio 0.76 per $1 \mathrm{ml} / \mathrm{kg}$ decrease, $95 \% \mathrm{Cl}$ 0.58 to $0.99, p=0.043$ ), after controlling for

Pneumocystis pneumonia, serum albumin, illness severity, gas exchange impairment and plateau pressure.

Conclusions: Lower tidal volume ventilation is independently associated with reduced mortality in HIV-infected patients with acute lung injury and respiratory failure.

Outcome-focused observational studies in critically ill HIV-infected patients admitted to the intensive care unit (ICU) have helped determine effective care practices since the beginning of the HIV epidemic. In particular, several reports from San Francisco General Hospital (SFGH) have documented how intensive care in this population has evolved since 1981. ${ }^{1-5}$ Because respiratory failure has been the leading cause of ICU admission and death throughout, several studies from our institution and from others have examined factors associated with mortality in patients with this presentation. Important factors have included greater severity of illness, lower serum albumin, the presence of Pneumocystis carinii pneumonia (PCP) and, among those with respiratory failure due to PCP, pneumothorax. ${ }^{235-8}$

Following the introduction of combination antiretroviral therapy (CART) in $1996,{ }^{9}$ studies at SFGH linked decreased ICU mortality to use of CART and to increases in non-AIDS admission diagnoses. ${ }^{58}$ At other institutions, the use of CART was also associated with a greater likelihood of a non-AIDS admission diagnosis, but not with decreases in ICU utilisation, respiratory failure, or in-hospital mortality. ${ }^{10} 11$ Finding no mortality benefit for CART, some authors have speculated that changing practices of ICU care rather than CART may explain decreased mortality over time. $^{12}$ Unfortunately, none of these studies has evaluated specific ICU therapies. ${ }^{13}$

One therapy associated with decreased ICU mortality in patients with respiratory failure is the use of low tidal volumes (VT) for mechanical ventilation in patients with acute lung injury (ALI) and the acute respiratory distress syndrome (ARDS), as described in the ARDS Network (ARDSNet) study in 2000. ${ }^{14}$ Because low VT ventilation is theoretically protective against barotrauma, ${ }^{15}$ it may be an important therapy for HIV-infected patients with severe respiratory failure due to PCP. Although no association between VT and barotrauma was observed in the ARDSNet study, pressure-volume relationships differ in HIVinfected patients with PCP and ALI compared with HIV-uninfected patients with ALI of different aetiologies, ${ }^{16}$ and the ARDSNet study included few HIV-infected patients. ${ }^{14}$ We therefore wished to examine whether low VT ventilation might be beneficial to HIV-infected patients with ALI.

To assess whether CART, low VT ventilation or other factors have an impact on mortality in this population, we assembled a retrospective cohort of all HIV-infected patients admitted to the ICU at SFGH between 1996 and 2004 with respiratory failure and ALI or ARDS (henceforth referred to aggregately as ALI). Our primary aim was to identify which therapies and clinical factors were associated with mortality in this population. A secondary aim was to compare mortality among these patients before and after introduction of a low VT ventilation protocol at SFGH in September 2000. Some patients, including all 74 enrolled in the pre-ARDSNet period, were included in previous publications, and some of the results have been previously reported in abstract form. ${ }^{581718}$ However, this is the first study to evaluate 
comprehensively the impact of low VT ventilation on mortality in HIV-infected patients admitted to the ICU with ALI.

\section{METHODS}

Clinical investigators (AM, AC, MB, LH) identified all HIVinfected patients with ALI admitted to the ICU at SFGH between 1996 and 2004 by searching the hospital's administrative database for patients with a discharge diagnosis of HIV disease (Ninth International Classification of Disease (ICD-9) code 042) and by merging this list with a registry of all patients with ALI. Investigators (AM, AC, LD, RK) reviewed medical records using standardised chart abstraction forms and identified study subjects from the subset of patients who required mechanical ventilation and met the American-European Consensus Criteria for ALI at any time during the ICU stay. ${ }^{19}$ For patients with multiple ICU visits within one hospitalisation, only the first ICU admission was considered. For one patient who developed ALI during two different hospitalisations (378 days apart), each episode was considered separately.

Predictor variables consisted of demographic characteristics including age, sex, race/ethnicity and HIV risk factor, clinical characteristics including HIV disease history (eg, PCP prophylaxis; CART, defined as use of $\geqslant 3$ antiretroviral agents from at least two drug classes on or after admission), aetiology of ALI (eg, microscopically confirmed PCP, clinically defined non-PCP pneumonia, clinically defined non-pulmonary cause of ALI) and height. Predictor variables also included laboratory data such as CD4+ $\mathrm{T}$ cell count (within 6 months), HIV RNA (within 6 months), haematocrit, serum albumin and lactate dehydrogenase $(\mathrm{LDH})$, arterial $\mathrm{pH}$, and arterial oxygen and carbon dioxide tensions. Ventilator parameters included mode of ventilation, expired tidal volume $(V T)$, respiratory rate $(R R)$, minute ventilation (VE), peak inspiratory pressure (PIP), endinspiratory plateau pressure (Pplat), mean airway pressure (Paw) and positive end-expiratory pressure (PEEP). Mechanical ventilation variables were recorded at the first ventilator check (which included arterial blood gas measurement and recording of static lung mechanics) after intubation on day 1 and on subsequent days at the time of the ventilator check closest to midday. Because ventilator settings on initiation of mechanical ventilation may not reflect the subsequent treatment strategy or mechanics, and because individual ventilator checks may record outlying values, we averaged all ventilator measurements over the first 3 days after the onset of ALI to increase accuracy and precision. Measures of illness severity at onset of ALI, including the Acute Physiology and Chronic Health Evaluation (APACHE-II) score, ${ }^{20}$ the Severe Acute Physiology (SAPS-II) score $^{21}$ and the Lung Injury Score (LIS), ${ }^{22}$ were calculated from abstracted variables. In addition, we calculated the following variables: compression-corrected VT (VT $-2.5 \mathrm{ml} / \mathrm{cm} \mathrm{H}_{2} \mathrm{O} \times$ [PIP - PEEP]), quasi-static respiratory system compliance (Crs $=\mathrm{VT} /[$ Pplat $-\mathrm{PEEP}])$, ratio of arterial oxygen tension to inspired oxygen concentration $\left(\mathrm{PaO}_{2} / \mathrm{FiO}_{2}\right)$, oxygenation index $\left(\mathrm{O}_{2}\right.$ index $\left.=\left[\mathrm{FiO}_{2} \times \mathrm{Paw}\right] / \mathrm{PaO}_{2}\right)$ and ideal body weight. ${ }^{23}$ The principal outcome variable was in-hospital mortality within 3 months.

\section{Data analysis}

Data were single-entered but subjected to internal logic and formatting checks. All analyses were performed using STATA 9.0 (Stata Corporation, College Station, TX, USA) with significance specified in reference to a two-tailed type I error ( $p$ value $)<0.05$. Bivariate analyses were performed using the $\chi^{2}$ test or Fisher exact test for dichotomous variables and the $t$ test or Mann-Whitney test for continuous variables.

For the secondary aim of the study-comparing mortality before and after the ARDS Network protocol was introduced at SFGH on 1 September 2000-we divided the dataset into two time periods. (Henceforth, we explicitly use the term "period" to avoid confusion with the "eras" described in the previous articles from this institution. We refer to the block of time from January 1996 to August 2000 as "the pre-ARDSNet period" or "the earlier period" and the block of time from September 2000 to December 2004 as "the post-ARDSNet period" or "the later period".) The sample size resulted from our a priori decision to limit the analysis to these 9 years.

Unlike the ARDSNet studies but similar to other studies of low VT ventilation, ${ }^{24}$ the ARDSNet Protocol at SFGH allows use of pressure-limited, volume-targeted ventilator modes to permit patient control over inspiratory flow. Otherwise, the ARDS Network protocol in use at SFGH follows exactly that outlined in the original ARDSNet study, ${ }^{14}$ targeting tidal volumes of 4-6 $\mathrm{ml} / \mathrm{kg}$ ideal body weight to maintain plateau pressures $<30 \mathrm{~cm} \mathrm{H}_{2} \mathrm{O}$.

Multivariate logistic regression analysis using backward selection was performed to assess whether demographic, clinical, laboratory and ventilator predictors or measures of illness severity and gas exchange impairment were associated with in-hospital mortality, and to control for potential confounders of the relationship between the predictors of primary interest (VT and CART) and in-hospital mortality. Because of the small sample size, we chose an inclusive cut-off $(p<0.2)$ for the empirical level of significance at which we would retain variables. We also included predictor variables if they altered by $10 \%$ or more the logit of any predictor variable that was also associated with the outcome at $p<0.2 .{ }^{25}$ Because PIP, Pplat, PEEP and LIS were highly correlated, we selected Pplat to represent these variables since it has the greatest face validity for measuring lung distension in ALI. Similarly, $\mathrm{PaO}_{2} /$ $\mathrm{FiO}_{2}$ was chosen to represent worst $\mathrm{PaO}_{2} / \mathrm{FiO}_{2}$ and $\mathrm{O}_{2}$ index because it is clinically familiar. The SAPS-II score was chosen in preference to the APACHE-II score because it better fit the assumptions of the logistic model. In addition, the variables serum albumin, PCP, CART use and VT (adjusted for ideal body weight) were included in the model because of high face validity

Table 1 Demographic and clinical characteristics of 148 HIV-infected patients with $\mathrm{ALI}$ in the ICU

\begin{tabular}{llll}
\hline & $\begin{array}{l}\text { Pre-ARDSNet } \\
\text { period } \\
\mathbf{1 9 9 6 - 2 0 0 0} \\
(\mathbf{n}=\mathbf{7 4 )}\end{array}$ & $\begin{array}{l}\text { Post-ARDSNet } \\
\text { period } \\
\text { Chana-2004 } \\
\text { (n= 74) }\end{array}$ & p Value \\
\hline Mean (SD) age (years) & $41.8(7.8)$ & $42.4(8.6)$ & 0.68 \\
Male sex (\%) & $60(81)$ & $51(74)$ & 0.30 \\
Caucasian ethnicity (\%) & $37(52)$ & $36(49)$ & 0.68 \\
MSM HIV risk factor (\%) & $25(34)$ & $24(32)$ & 0.86 \\
CART on admission or while in & $20(27)$ & $28(38)$ & 0.16 \\
ICU (\%) & & $26(36)$ & 0.23 \\
PCP prophylaxis on admission (\%) & $31(46)$ & $14(19)$ & 0.42 \\
Previous PCP (\%) & $18(25)$ & $63(85)$ & 0.21 \\
Pulmonary cause of ALI (\%) & $57(77)$ & $27(36)$ & 0.60 \\
PCP (\%) & $24(32)$ & $16(22)$ & 0.20 \\
Pneumothorax (\%) & $10(14)$ & \\
\hline
\end{tabular}

*Up to 24 observations missing per era, as for HIV risk factor. ALI, acute lung injury; ARDSNet, Acute Respiratory Distress Syndrome Network study; CART, combination antiretroviral therapy; ICU, intensive care unit; PCP, Pneumocystis pneumonia; MSM, men having sex with men; SD, standard deviation. 
and prespecified importance to the research question. To control for confounding due to changes in ICU outcomes over time, we included a dichotomous variable for care in the period after introduction of the ARDSNet ventilation protocol compared with care in the prior period. Interaction terms to test the hypotheses that having PCP or a low CD4 count might enhance any mortality benefit of CART, or that lower VT might be effective in patients with PCP or in patients cared for in the post-ARDSNet period, were also evaluated. The final model was assessed for goodness of fit using the Hosmer-Lemeshow test and for omitted covariates and model misspecification using the link test. ${ }^{24}$

\section{RESULTS}

Of the 685 consecutive HIV-infected adults admitted to the ICU at SFGH between 1996 and 2004, 148 (22\%) had ALI. Half were admitted before the introduction of the ARDSNet protocol and half after. Of the 148 patients with ALI, 132 (89\%) had ARDS, the most severe form of ALI. The principal causes of ALI were PCP (34\%), non-PCP pneumonia (38\%), sepsis (15\%) and aspiration pneumonitis (7\%). As a proportion of all ICU admissions for HIV-infected persons, ALI was more common in the post-ARDSNet period (25\%) than in the pre-ARDSNet period $(19 \%)(p=0.045)$.

In-hospital mortality in the post-ARDSNet period (54\%) was similar to that in the pre-ARDSNet period (59\%), but the wide confidence intervals surrounding the point estimate of the risk difference prevented exclusion of a clinically important increase or decrease in mortality (risk difference $-5.4 \%$, 95\% confidence interval (CI) $-21 \%$ to $11 \%, p=0.51)$. ICU mortality was slightly lower, $51 \%$ in the earlier period and $46 \%$ in the later period, with the same risk difference (risk difference $-5.4 \%$, $95 \% \mathrm{CI}-21 \%$ to $11 \%, \mathrm{p}=0.51)$. From the earlier to the later period there were no significant differences in median days of mechanical ventilation ( 8 vs $8, p=0.39$ ), days in ICU (9 vs 8.5, $\mathrm{p}=0.93$ ) or days in hospital (20 vs $19, \mathrm{p}=0.78$ ).

The demographic, clinical and laboratory characteristics of persons with ALI were similar between the two periods (tables 1 and 2). The proportions with PCP did not differ between the

Table 2 Laboratory parameters and illness severity of 148 HIV-infected patients with ALI in the ICU

\begin{tabular}{|c|c|c|c|}
\hline Characteristic* & $\begin{array}{l}\text { Pre-ARDSNet } \\
\text { period } \\
1996-2000 \\
(\mathrm{n}=74)\end{array}$ & $\begin{array}{l}\text { Post-ARDSNet } \\
\text { period } \\
2000-2004 \\
(n=74)\end{array}$ & p Value \\
\hline $\begin{array}{l}\text { Median (IQR) CD4+ T cell count } \\
\text { (cells/ } / \mu \text { l) }\end{array}$ & $65(17-230)$ & $67(18-188)$ & 0.91 \\
\hline $\begin{array}{l}\text { Median (IQR) } \log _{10} \text { HIV RNA } \\
\text { (copies/ml) }\end{array}$ & $4.9(4.2-5.3)$ & $5.2(4.1-5.5)$ & 0.20 \\
\hline Mean (SD) albumin (g/dl) & $2.28(0.54)$ & $2.40(0.72)$ & 0.28 \\
\hline Median (IOR) LDH (IU) & $365(255-517)$ & $366(239-576)$ & 0.96 \\
\hline Mean $\mathrm{pH} \dagger<7.25(\%)$ & $23(31)$ & $24(32)$ & 0.86 \\
\hline Mean (SD) $\mathrm{Paco}_{2} \dagger(\mathrm{mm} \mathrm{Hg})$ & $39(10)$ & $40(8.9)$ & 0.43 \\
\hline Mean (SD) $\mathrm{PaO}_{2} \dagger(\mathrm{mm} \mathrm{Hg})$ & $93(24)$ & $93(23)$ & 0.88 \\
\hline Mean (SD) APACHE-II score & $24(6.6)$ & $23(5.9)$ & 0.19 \\
\hline Mean (SD) SAPS-II score & $55(18)$ & $53(14)$ & 0.53 \\
\hline LIS $\geqslant 2.75(\%)$ & $29(42)$ & $51(69)$ & 0.001 \\
\hline
\end{tabular}

*Up to 35 observations missing, as for HIV RNA.

†Physiological variables averaged over days 1-3.

ALI, acute lung injury; ARDSNet, Acute Respiratory Distress Syndrome Network study; APACHE-II, Acute Physiology and Chronic Health Evaluation II; ICU, intensive care unit; IQR, interquartile range; LDH, lactate dehydrogenase; LIS, Lung Injury Score; $\mathrm{PaCO}_{2}$, carbon dioxide tension; $\mathrm{PaO}_{2}$, arterial oxygen tension; SAPS-II, Severe Acute Physiology Score II; SD standard deviation. two periods, nor did the proportion suffering pneumothorax change significantly after introduction of the ARDSNet protocol. A similar proportion of patients received CART in the later period $(38 \%)$ as in the earlier period $(27 \%)(p=0.16)$, and CD4 counts and HIV RNA levels were comparable in the two periods. Although the median CD4 count did not differ between those taking and not taking CART (78.5 vs 60, $p=0.78$ ), log HIV RNA was lower in those taking CART than in those not taking CART (4.7 vs 5.2, $\mathrm{p}=0.005$ ).

The use of pressure-regulated modes of ventilation at any time during the first 3 days in the ICU was more common in the post-ARDSNet period (39\%) than in the pre-ARDSNet period $(22 \%)(p=0.020)$, a difference reflecting the increased use of dual modes of ventilation in the later period (table 3 ). Mean VT, corrected for ideal body weight and averaged over days $1-3$, was lower in the later period than in the earlier period $(6.6 \mathrm{ml} / \mathrm{kg}$ vs $9.1 \mathrm{ml} / \mathrm{kg}, \mathrm{p}<0.001)$. Although the respiratory rate was higher $(p<0.001)$ and VE lower $(p=0.002)$ in the later period than in the earlier period, the number of patients with moderate or severe acidaemia $(\mathrm{pH}<7.25)$ was similar $(31 \%$ in the pre-ARDSNet period vs $32 \%$ in the post-ARDSNet period, $\mathrm{p}=0.86$ ) and mean $\mathrm{PaCO}_{2}$ was stable between periods (39 vs 40, $\mathrm{p}=0.43$ ). Assuming $\mathrm{CO}_{2}$ production remained stable from the earlier period to the later period, this finding suggests that wasted ventilation (dead-space fraction) declined in the postARDSNet period.

Median Pplat tended to be lower $(p=0.082)$ and median PEEP was higher $(p=0.037)$ after introduction of the ARDSNet protocol at SFGH. Overall severity of lung injury, as measured by LIS, was substantially worse in the later period than in the earlier period, primarily because respiratory system compliance was lower in the later period $\left(28 \mathrm{ml} / \mathrm{cm} \mathrm{H}_{2} \mathrm{O}\right.$ post-ARDSNet protocol vs $36 \mathrm{ml} / \mathrm{cm} \mathrm{H}_{2} \mathrm{O}$ pre-ARDSNet protocol, $\mathrm{p}<0.001$ ). Mean plateau pressure was higher in those who developed pneumothoraces than in those who did not $\left(29\right.$ vs $26 \mathrm{~cm} \mathrm{H}_{2} \mathrm{O}$, $\mathrm{p}=0.054)$, although compliance and other static pressures did not differ ( $p \geqslant 0.19$ for all comparisons). Mean VT was lower among those with PCP than among those with other causes of ALI (7.0 vs $8.2 \mathrm{ml} / \mathrm{kg}, \mathrm{p}=0.002)$.

In unadjusted analyses, most HIV-related variables-including CD4 count, HIV RNA, use of CART, use of PCP prophylaxis and presence of PCP-were not clearly associated with death (table 4). In contrast, higher levels of non-HIV-related variables_PIP and Pplat-were clearly associated with mortality. Other ALI-related variables, including higher VT, higher PEEP and higher LIS, showed trends towards increased mortality. Markers of severity of illness, including lower serum albumin; higher APACHE-II and higher SAPS-II scores; lower $\mathrm{PaO}_{2} / \mathrm{FiO}_{2}$ and lower worst $\mathrm{PaO}_{2} / \mathrm{FiO}_{2}$; and higher $\mathrm{O}_{2}$ index were also significantly associated with death.

To construct a causal multivariate model we included variables for severity of illness, static lung mechanics, $V_{T}$ and oxygenation on the grounds of their empirical associations with both period and mortality at $p<0.2$. We also included the prespecified variables CART, PCP, albumin and period on the grounds of face validity. The final model showed ALI-related variables to be more strongly associated with mortality than HIV-related factors (table 4). After controlling for period of ICU care, $\mathrm{PaO}_{2} / \mathrm{FiO}_{2}$ and use of CART, the presence of PCP, lower serum albumin, higher SAPS-II score and higher Pplat were all independently associated with increased mortality. Lower VT was independently associated with decreased mortality (adjusted odds ratio 0.76 per $1 \mathrm{ml} / \mathrm{kg}$ decrease, $95 \%$ CI 0.58 to $0.99, p=0.043$ ). During stepwise removal of predictors from the 
Table 3 Ventilation parameters for 148 HIV-infected patients with ALI in ICU

\begin{tabular}{|c|c|c|c|}
\hline Characteristic* & $\begin{array}{l}\text { Pre-ARDSNet } \\
\text { period } \\
1996-2000 \\
(n=74)\end{array}$ & $\begin{array}{l}\text { Post- } \\
\text { ARDSNet } \\
\text { period } \\
2000-2004 \\
(n=74)\end{array}$ & p Value \\
\hline Pressure-regulated ventilation (\%) & $16(22)$ & $29(39)$ & 0.020 \\
\hline Mean (SD) $\mathrm{V} T_{\uparrow} \dagger(\mathrm{ml} / \mathrm{kg})$ & $9.1(2.0)$ & $6.6(1.5)$ & $<0.001$ \\
\hline Mean (SD) $R R \uparrow$ (breaths/min) & $22(5.8)$ & $25(6.2)$ & $<0.001$ \\
\hline Median (IOR) VE† (I/min) & $14(11-17)$ & $11(10-14)$ & 0.002 \\
\hline Mean (SD) $\mathrm{PIP} \dagger\left(\mathrm{cm} \mathrm{H} \mathrm{H}_{2} \mathrm{O}\right)$ & $36(8.2)$ & $35(7.5)$ & 0.37 \\
\hline Median (IOR) Pplat $\uparrow\left(\mathrm{cm} \mathrm{H} \mathrm{H}_{2} \mathrm{O}\right)$ & $29(23-31)$ & $26(23-29)$ & 0.082 \\
\hline Median (IOR) Paw† (cm H $\left.\mathrm{H}_{2} \mathrm{O}\right)$ & $15(12-18)$ & $17(13-19)$ & 0.098 \\
\hline Median (IOR) PEEP $\dagger\left(\mathrm{cm} \mathrm{H}_{2} \mathrm{O}\right)$ & $7.0(5.0-9.3)$ & $8.3(5.0-10)$ & 0.037 \\
\hline Mean (SD) Crs $\dagger\left(\mathrm{ml} / \mathrm{cm} \mathrm{H}_{2} \mathrm{O}\right)$ & $36(11)$ & $28(11)$ & $<0.001$ \\
\hline Median (IQR) $\mathrm{PaO}_{2} / \mathrm{FiO}_{2} \uparrow(\mathrm{mm} \mathrm{Hg})$ & $144(111-193)$ & $137(103-179)$ & 0.49 \\
\hline $\begin{array}{l}\text { Median (IOR) worst } \mathrm{PaO}_{2} / \mathrm{FiO}_{2} \dagger \\
(\mathrm{mm} \mathrm{Hg})\end{array}$ & $125(84-174)$ & $120(90-159)$ & 0.88 \\
\hline $\begin{array}{l}\text { Median (IOR) } \mathrm{O}_{2} \text { index† }(\mathrm{mm} \mathrm{Hg} / \\
\left.\mathrm{cm} \mathrm{H} \mathrm{H}_{2} \mathrm{O}\right)\end{array}$ & $10(6.5-16)$ & $12(8.8-16)$ & 0.20 \\
\hline
\end{tabular}

*Up to 11 observations missing, as for VT and PIP. †Physiological variables averaged over days $1-3$

ALI, acute lung injury; ARDSNet, Acute Respiratory Distress Syndrome Network study; Crs, respiratory system compliance; $\mathrm{Fi}_{2}$, fractional inspired oxygen concentration; ICU, intensive care unit; IQR, interquartile range; $\mathrm{O}_{2}$, oxygenation; $\mathrm{PaO}_{2}$, arterial oxygen tension; PIP, peak inspiratory pressure; Pplat, end-inspiratory plateau pressure; Paw, mean airway pressure; PEEP, positive end-expiratory pressure; $\mathrm{RR}$, respiratory rate; $\mathrm{SD}$, standard deviation; $\mathrm{VE}_{\mathrm{E}}$ minute ventilation; $\mathrm{VT}$, tidal volume.

final model, PCP was the principal confounder suppressing the association between lower VT and mortality. CART was not independently associated with mortality $(p=0.82)$, and excluding it from the model had no effect on the other variables. The same associations were observed with alternative model building approaches, including stepwise forward selection and automated regression techniques.

After including interaction terms in the model, there was a strong association between CART and decreased mortality among those with PCP (adjusted odds ratio 0.14, 95\% CI 0.024 to $0.83, p=0.031)$. There was no interaction between CART and CD4 count, between lower VT and PCP, or between lower $\mathrm{VT}$ and period of enrolment ( $p>0.10$ for each interaction).

Excluding influential data points did not significantly alter the overall model. There was no evidence that quadratic terms or additional covariates would improve the model ( $p=0.15$ by the Wald link specification test), which appeared robust across all strata ( $p=0.64$ by the Hosmer-Lemeshow statistic).

\section{DISCUSSION}

Our study is the first to show that survival among HIV-infected patients with respiratory failure and ALI is associated with a specific non-HIV-related ICU therapy, lower VT ventilation. CART was associated with greatly reduced odds of death only in patients with PCP, but this diagnosis was a rare cause of respiratory failure in the ICU. The decrease in mortality that we observed after introduction of the ARDSNet protocol did not reach statistical significance, probably because of insufficient sample size. Measuring VT as a continuous variable, however, gave the study adequate power to show that lower VT was associated with significantly reduced odds of mortality.

This study updates previous studies of critically ill HIVinfected patients by showing that CART may benefit some HIV-infected patients with respiratory failure (eg, those with
PCP) but not necessarily all. While one study reported a longterm survival benefit from use of CART in critically ill HIVinfected patients, ${ }^{10}$ most studies have reported no increase in survival to discharge. ${ }^{10} 1126$ A study at a university hospital in the UK also demonstrated no survival benefit to CART in patients with PCP. ${ }^{12}$ Only studies from SFGH, including those with and without PCP, have shown a CART-associated decrease in hospital deaths. ${ }^{5}$ Patient selection is one possible reason for these discrepancies. Almost a quarter of those on CART in our study had it initiated after hospital admission, a practice which is uncommon among other studies reporting this information. ${ }^{11}{ }^{12}$ In addition, all patients on CART in this study were using a regimen that included a protease inhibitor, an antiretroviral class which may have anti-Pneumocystis activity. ${ }^{27}$ Determining which of these factors accounts for the CARTassociated decrease in PCP mortality will require studies that either randomise patients or adjust for the propensity of clinicians to treat with CART.

This study confirms the previously reported independent associations with mortality of several clinical variables including illness severity, serum albumin and the presence of PCP. ${ }^{2}{ }^{4} \mathrm{To}$ previous data showing that non-HIV admission diagnoses are an important predictor of survival in HIV-infected patients in the ICU, ${ }^{5}$ this study adds the observation that one such diagnosis-ALI-is becoming more severe. Although the reasons for this finding are unclear, the greater frequency of severe LIS in the post-ARDSNet protocol period probably reflects lower Crs which has been associated with low VT ventilation, possibly through increased atelectasis, elastic recoil and patient-ventilator dyssynchrony. ${ }^{28} 29$

As the largest study of HIV-associated respiratory failure to date, this study provides specific physiological data associating the use of lower VT in ALI with decreased odds of in-hospital death. These results support previous findings that lower VT in ALI is associated with decreased mortality in clinical trial and practice settings, ${ }^{14}{ }^{17}$ and reinforce the recommendation that HIV-infected persons with ALI requiring mechanical ventilation receive low VT ventilation, ideally by protocol. ${ }^{30}$

Unlike previous PCP-focused studies of HIV-associated respiratory failure, ${ }^{78}$ pneumothorax was not associated with mortality in this study. Furthermore, as in other studies of ALI, ${ }^{31} 32$ barotrauma did not explain the mortality benefit of lower VT ventilation in this population. We observed a probable decrease in dead-space fraction from the early period to the later period, possibly due to a low VT-associated reduction in alveolar distension (as indicated by reduced Pplat). Given the known association between dead-space fraction and mortality in ARDS, ${ }^{33}$ this observation bears further investigation as a potential mechanism for decreased mortality in ALI.

This study shows that Pplat is independently associated with death in HIV-infected patients with ALI, as previously reported in HIV-negative patients. ${ }^{34}$ The association between lung mechanics and outcome in ALI probably depends on more than VT, however, since the association between Pplat and death persisted even after adjusting for its principal determinantsCrs, VT and PEEP. Although these adjustments do not account for the contribution of chest wall compliance to total elastic recoil, chest wall compliance is minimally disturbed in direct causes of ALI. ${ }^{16}{ }^{35}$ Since $80 \%$ of our patients with ALI had direct lung injury, primarily from pneumonia, Pplat is probably an accurate reflection of lung stress in this cohort. It has recently been shown that lung overdistension commonly occurs in ARDS despite the use of the ARDSNet protocol, and that a relatively higher mortality is associated with even modestly 
acted as significant confounders. Furthermore, no other ICU protocols were in systematic use at SFGH during the study period.

In summary, survival of critically ill HIV-infected patients with ALI and respiratory failure was associated with ventilation with lower VT. Among the subset of patients with PCP and ALI, use of CART on or after admission was strongly correlated with lower mortality. Even after adjustment for VT, lower Pplat was independently associated with a decrease in the odds of death in all patients studied. Future studies of outcomes in HIV-infected patients in the ICU should consider the interaction between HIV- and non-HIV-associated admission diagnoses and specific therapies in the ICU.

Acknowledgements: The authors thank the patients and staff of San Francisco General Hospital for their contributions to the data gathered for this study; Ms Alexandra Swartzman and Mr Anthony Cacatian for assistance in obtaining medical records; and Professor Peter Bacchetti for statistical advice.

Funding: NIH 5T32HL007185-30 (JLD), NIH P30AI027763-15 (JLD), NIH 1F32HL088990-01 (JLD), NIH K23HL072837 (AM),

NIH R01HL083461 (AM), NIH K24HL087713 (LH), NIH R01 HL090335 (LH).

Competing interests: None.

Ethics approval: The Committee on Human Research at the University of California, San Francisco approved the study protocol.

\section{REFERENCES}

1. Wachter RM, Luce JM, Turner J, et al. Intensive care of patients with the acquired immunodeficiency syndrome. Outcome and changing patterns of utilization. Am Rev Respir Dis 1986;134:891-6.

2. Wachter RM, Russi MB, Bloch DA, et al. Pneumocystis carinii pneumonia and respiratory failure in AIDS. Improved outcomes and increased use of intensive care units. Am Rev Respir Dis 1991;143:251-6.

3. Wachter RM, Luce JM, Safrin S, et al. Cost and outcome of intensive care for patients with AIDS, Pneumocystis carinii pneumonia, and severe respiratory failure. JAMA 1995;273:230-5.

4. Nickas G, Wachter RM. Outcomes of intensive care for patients with human immunodeficiency virus infection. Arch Intern Med 2000;160:541-7.

5. Morris A, Creasman J, Turner J, et al. Intensive care of human immunodeficiency virus-infected patients during the era of highly active antiretroviral therapy. Am J Respir Crit Care Med 2002;166:262-7.

6. Wachter RM, Luce JM. Respiratory failure from severe Pneumocystis carinii pneumonia. Entering the third era. Chest 1994:106:1313-5.

7. Bedos JP, Dumoulin JL, Gachot B, et al. Pneumocystis carinii pneumonia requiring intensive care management: survival and prognostic study in 110 patients with human immunodeficiency virus. Crit Care Med 1999:27:1109-15.

8. Morris A, Wachter RM, Luce J, et al. Improved survival with highly active antiretroviral therapy in HIV-infected patients with severe Pneumocystis carinii pneumonia. AIDS 2003;17:73-80.

9. Collier AC, Coombs RW, Schoenfeld DA, et al. Treatment of human immunodeficiency virus infection with saquinavir, zidovudine, and zalcitabine. AIDS Clinical Trials Group. N Engl J Med 1996;334:1011-7.

10. Casalino E, Wolff M, Ravaud $P$, et al. Impact of HAART advent on admission patterns and survival in HIV-infected patients admitted to an intensive care unit. AIDS 2004;18:1429-33.

11. Dickson SJ, Batson S, Copas AJ, et al. Survival of HIV-infected patients in the intensive care unit in the era of highly active antiretroviral therapy. Thorax 2007;62:964-8.

12. Miller RF, Allen E, Copas A, et al. Improved survival for HIV infected patients with severe Pneumocystis jirovecii pneumonia is independent of highly active antiretroviral therapy. Thorax 2006;61:716-21.
13. Narasimhan $\mathbf{M}$, Posner AJ, DePalo VA, et al. Intensive care in patients with HIV infection in the era of highly active antiretroviral therapy. Chest 2004;125:1800-4.

14. The Acute Respiratory Distress Syndrome Network. Ventilation with lower tidal volumes as compared with traditional tidal volumes for acute lung injury and the acute respiratory distress syndrome. N Engl J Med 2000;342:1301-8.

15. Gammon RB, Shin MS, Buchalter SE. Pulmonary barotrauma in mechanical ventilation. Patterns and risk factors. Chest 1992;102:568-72.

16. D'Angelo E, Calderini E, Robatto FM, et al. Lung and chest wall mechanics in patients with acquired immunodeficiency syndrome and severe Pneumocystis carinii pneumonia. Eur Respir J 1997;10:2343-50.

17. Kallet RH, Jasmer RM, Pittet JF, et al. Clinical implementation of the ARDS network protocol is associated with reduced hospital mortality compared with historical controls. Crit Care Med 2005;33:925-9.

18. Davis JL, Morris AM, Powell K, et al. Intensive care of HIV-infected patients with pneumocystis pneumonia in the era of combination antiretroviral therapy (CART) [abstract]. American Thoracic Society International Conference; San Francisco, 2007.

19. Bernard GR, Artigas A, Brigham KL, et al. The American-European Consensus Conference on ARDS. Definitions, mechanisms, relevant outcomes, and clinical trial coordination. Am J Respir Crit Care Med 1994:149(3 Pt 1):818-24.

20. Knaus WA, Draper EA, Wagner DP, et al. APACHE II: a severity of disease classification system. Crit Care Med 1985:13:818-29.

21. Le Gall JR, Lemeshow S, Saulnier F. A new Simplified Acute Physiology Score (SAPS II) based on a European/North American multicenter study. JAMA 1993:270:2957-63.

22. Murray JF, Matthay MA, Luce JM, et al. An expanded definition of the adult respiratory distress syndrome. Am Rev Respir Dis 1988;138:720-3.

23. Devine BJ. Gentamicin therapy. Drug Intell Clin Pharm 1974:8:650-5.

24. Amato MB, Barbas CS, Medeiros DM, et al. Effect of a protective-ventilation strategy on mortality in the acute respiratory distress syndrome. N Engl J Med 1998;338:347-54

25. Vittinghoff E. Regression methods in biostatistics: linear, logistic, survival, and repeated measures models. New York: Springer, 2005.

26. Khouli H, Afrasiabi A, Shibli M, et al. Outcome of critically ill human immunodeficiency virus-infected patients in the era of highly active antiretroviral therapy. J Intensive Care Med 2005;20:279-85.

27. Atzori C, Angeli E, Mainini A, et al. In vitro activity of human immunodeficiency virus protease inhibitors against Pneumocystis carinii. J Infect Dis 2000;181:1629-34.

28. Suter PM, Fairley HB, Isenberg MD. Effect of tidal volume and positive endexpiratory pressure on compliance during mechanical ventilation. Chest 1978;73:158-62.

29. Kallet RH, Luce JM. Detection of patient-ventilator asynchrony during low tidal volume ventilation, using ventilator waveform graphics. Respir Care 2002;47:183-5.

30. Huang L, Quartin A, Jones D, et al. Intensive care of patients with HIV infection. N Engl J Med 2006:355:173-81.

31. Gammon RB, Shin MS, Groves RH Jr, et al. Clinical risk factors for pulmonary barotrauma: a multivariate analysis. Am J Respir Crit Care Med 1995;152/4 Pt 1):1235-40.

32. Stewart TE, Meade MO, Cook DJ, et al. Evaluation of a ventilation strategy to prevent barotrauma in patients at high risk for acute respiratory distress syndrome. Pressure- and Volume-Limited Ventilation Strategy Group. N Engl J Med 1998;338:355-61.

33. Nuckton TJ, Alonso JA, Kallet RH, et al. Pulmonary dead-space fraction as a risk factor for death in the acute respiratory distress syndrome. N Engl J Med 2002:346:1281-6.

34. Hager DN, Krishnan JA, Hayden DL, et al. Tidal volume reduction in patients with acute lung injury when plateau pressures are not high. Am J Respir Crit Care Med 2005:172:1241-5.

35. Gattinoni L, Pelosi P, Suter PM, et al. Acute respiratory distress syndrome caused by pulmonary and extrapulmonary disease. Different syndromes? Am J Respir Crit Care Med 1998;158:3-11.

36. Terragni PP, Rosboch G, Tealdi A, et al. Tidal hyperinflation during low tidal volume ventilation in acute respiratory distress syndrome. Am J Respir Crit Care Med 2007:175:160-6.

37. Gattinoni L, Vagginelli F, Chiumello D, et al. Physiologic rationale for ventilator setting in acute lung injury/acute respiratory distress syndrome patients. Crit Care Med 2003;31 (4 Suppl):S300-4 